\title{
DER 1. THORNER FRIEDEN (1411) IM SPIEGEL DER SÖLDNERFRAGE
}

\section{目} as Jahr 2010 hat anlässlich der 600-Jahrfeier der berühmten Schlacht bei Tannenberg-Grunwald-Žalgiris eine unüberschaubare Fülle von Literatur zum Thema des „Großen Krieges" zwischen Polen-Litauen und dem Deutschen Orden in Preußen in den Jahren 1409 bis 1411 zur Folge gehabt. Ich brauche nur auf die mehr als 1150 Titel umfassende „Bibliografia grunwaldzka“ hinzuweisen, die in der Wojewodschaftsbibliothek in Olsztyn (Allenstein) für die Jahre 1990 bis 2010 erstellt wurde und trotz der umfangreichen Arbeit der drei Bearbeiterinnen keineswegs vollständig ist. ${ }^{1}$ Viele Veröffentlichungen sind publizistischer Natur und werden bald vergessen sein, aber es gibt auch wissenschaftliche Werke von bestehendem Wert. Von solchen, ich möchte sagen „Leuchttürmen“, die aus dem Meer der Publizistik und der Populärliteratur herausragen und in Polen erschienen sind, will ich hier nur drei Beispiele erwähnen. Ich denke zunächst an den zweibändigen und 4 Kilogramm schweren Grunwald-Ausstellungskatalog des Königlichen Schlosses auf dem Wawel mit vielen Beiträgen, farbigen Abbildungen und einer Bibliographie mit rund 1450 Titeln. ${ }^{2}$ Von großer Bedeutung ist ferner das fast 850 Seiten umfassende Buch über den genannten Krieg, das vom Schlossmuseum Malbork (Marienburg) herausgegeben und von drei Historikern der Nicolaus-Copernicus-Universität und einem weiteren Kollegen von der Universität Gdańsk (Danzig) verfasst wurde. ${ }^{3}$ Dieses Werk wird durch seine Quellennähe und gründliche Auswertung der Archivalien des Deutschen Ordens im Geheimen

1 A. Romulewicz, A. Wysocka, S. Białecka, Bibliografia grunwaldzka za lata 1990-2010, Olsztyn 2010. Eine ergänzende Bibliographie ist zur Zeit in Arbeit.

2 Na znak świetnego zwycięstwa w sześśsetna rocznice bitwy pod Grunwaldem. Katalog wystawy 15 lipca - 30 września 2010, Kraków 2010, Bd. I: Studia; Bd. II: Noty katalogowe; Bibliographie in Bd. II, S. 403-432.

3 S. Jóźwiak, K. Kwiatkowski, A. Szweda, S. Szybkowski, Wojna Polski i Litwy z zakonem krzyżackim w latach 1409-1411, Malbork 2010; Bibliographie auf S. 766-813. 
Staatsarchiv Preußischer Kulturbesitz in Berlin, namentlich des Ordensbriefarchivs (OBA) ${ }^{4}$, ein Standardwerk bleiben. Hier umfasst das Quellen- und Literaturverzeichnis 48 Seiten. Schließlich sei auch der Ausstellungskatalog „Grunwald - 600 lat chwaty“ (Grunwald - 600 Jahre Ehre) des Hauptarchivs Alter Akten in Warszawa (Warschau) genannt. ${ }^{5}$ Darin finden sich unter anderm hervorragende Abbildungen von Vertragsurkunden und Siegeln, beispielsweise von der Urkunde des Hochmeisters Heinrich von Plauen über den 1. Thorner Frieden am 1. Februar 1411.6 Am 14. Mai 2011 wurde übrigens in Kooperation mit dem Deutschen Historischen Institut Warschau eine Tagung im Archiwum Główne Akt Dawnych (AGAD; dt. Hauptarchiv Alter Akten) zum Thema „Der erste Thorner Frieden 1411 und seine Bedeutung für die Geschichte Zentral- und Ostmitteleuropas" veranstaltet. Die dort gehaltenen Vorträge sind als Veröffentlichung in der Reihe „Miscellanea Historica Archivistica“ des AGAD vorgesehen.

Als ich vor nunmehr drei Jahren auf einer Tagung in Bytów (dt. Bütow) im Oktober 2008 von Professor Czaja gebeten wurde, einen Vortrag mit Bezug auf den 1. Thorner Frieden auf dieser Ordines Militares-Tagung in Torun (Thorn) zu halten, war die bevorstehende Fülle und Intensität der polnischen Forschung auch zu diesem Thema nicht vorauszusehen.

Trotz der umfassenden Literatur gibt es bisher keine Untersuchung über den Friedensschluss in Bezug auf die Söldnerfrage im Ordensland und in Polen, weder in der älteren Forschung, noch in den jüngsten Beiträgen der Kollegen Jóźwiak, Kwiatkowski, Szweda, Szybkowski und Grabowski. Sie befassen sich mit anderen Aspekten der Thematik. ${ }^{8}$

Ausgangspunkt meiner Überlegungen sind meine neuen Forschungsergebnisse über die Anwerbung von Söldnern vor der Schlacht bei Tannenberg-Grunwald,

4 Geheimes Staatsarchiv Preußischer Kulturbesitz, Berlin, XX. Hauptabteilung Historisches Staatsarchiv Königsberg, Ordensbriefarchiv (GStA PK, XX. HA StA. Kbg., OBA). Im Folgenden wird der Bestand nur als OBA mit der betreffenden Nummer des Briefes zitiert.

5 Grunwald. 600 lat chwaty. Katalog wystawy, hrsg. v. J. Grabowski, Warszawa 2010.

6 Ebd., S. 132-133.

7 In Bd. XIX unter der Hauptredaktion von Jacek Krochmal. Von den Vorträgen mögen hier vier genannt werden: S. Flemmig, Der Erste Thorner Friede in den Beziehungen des Deutschen Ordens zum Reich; S. Szybkowski, Die personelle Umgebung von König Wtadystaw Jagietto während der Januarverhandlungen mit dem Deutschen Orden im Jahr 1411; A. Szweda, Wie wurden die im 1. Thorner Frieden festgehaltenen Vertragsbedingungen im weiteren Verlauf der Beziehungen zwischen Polen und dem Deutschen Orden eingehalten?; J. Tandecki, Die preußischen Städte vor und nach dem Thorner Frieden vom 1. Feb. 1411.

8 Siehe dazu die entsprechenden Kapitel und Abschnitte in dem oben genannten Buch Wojna Polski i Litwy (wie Anm. 3), vor allem Kapitel VI, S. 694-747; sowie, was Janusz Grabowski betrifft, S. 49-56, 132 in dem Ausstellungskatalog Grunwald. 600 lat chwaty (wie Anm. 5). 
die ich während eines Vortrags auf der genannten Konferenz in Bytów im Oktober 2008 vorstellte. ${ }^{9}$ Es ging dabei u. a. um meine Neudatierung einer wichtigen Quelle im Ordensbriefarchiv (OBA 1250), die bis dahin in der Forschung immer auf den 26. Januar 1410 datiert worden war, ${ }^{10}$ die jedoch erst fünf Wochen vor der Schlacht, am 8. Juni, entstanden ist ${ }^{11}$. Diese bedeutsame Neudatierung hat inzwischen auch in der polnischen Geschichtsschreibung einen festen Platz gefunden. ${ }^{12}$ Sie hat als „Kettenreaktion“ zu weiteren wichtigen Untersuchungen aus meiner Feder in Bezug auf die Politik und die Söldnerwerbungen des Deutschen Ordens und Polen-Litauens während der letzten Monate und Wochen vor der Schlacht geführt. ${ }^{13}$ Auch jene Ergebnisse sind von den polnischen Kollegen voll angenommen worden. ${ }^{14}$ Ich will sie hier nicht im Einzelnen wiederholen, möchte jedoch erneut die Bedeutung des vom Hochmeister Ulrich von Jungingen Mitte

9 Der Vortrag erschien zwei Jahre später im Druck. S. Ekdahl, Die Söldnerwerbungen des Deutschen Ordens für einen geplanten Angriff auf Polen am 1. Juni 1410. Ein Beitrag zur Vorgeschichte der Schlacht bei Tannenberg, in: Beiträge zur Militärgeschichte des Preußenlandes von der Ordenszeit bis zum Zeitalter der Weltkriege (Tagungsberichte der Historischen Kommission für ost- und westpreußische Landesforschung 25), hrsg. v. B. Jähnig, Marburg 2010, S. 89-102.

10 Siehe beispielsweise S. Jóźwiak, Wywiad i kontrwywiad w państwie zakonu krzyżackiego w Prusach. Studium nad sposobami pozyskiwania i wykorzystywaniem poufnych informacji w późnym średniowieczu, Malbork 2004, S. 19, 164, 197, 203, 241.

11 Ekdahl, Die Söldnerwerbungen (wie Anm. 9), S. 97-99.

12 Siehe beispielsweise Sławomir Jóźwiak in Wojna Polski i Litwy (wie Anm. 3), S. 221 mit der langen Anm. 182, die weitere Argumente für die Richtigkeit der Neudatierung bringt; ders., Spionage zur Zeit des polnisch-litauischen Krieges gegen den Deutschen Orden 1409-1411, in: Tannenberg-Grunwald-Žalgiris 1410: Krieg und Frieden im späten Mittelalter (Deutsches Historisches Institut Warschau. Quellen und Studien 26), hrsg. v. W. Paravicini, R. Petrauskas, G. Vercamer, Wiesbaden 2012, S. 191-197, hier 195.

13 S. Ekdahl, Diplomatie und Söldnerwerbung vor der Schlacht bei Žalgiris, in: Lietuvos istorijos studijos 25 (2010), S. 48-61; ders., Nabor najemnikow pjered Grjunwaldskoi bitwoi $1410 \mathrm{~g}$. w kontjeksti politiki i diplomatii epochi [Die Söldnerwerbung vor der Grunwald-Schlacht 1410 im politischen und diplomatischen Kontext der Epoche], Studia Slavica et Balcanica Petropolitana 2010, 2, S. 17-24; ders., Politics, Diplomacy and the Recruitment of Mercenaries before the Battle of Tannenberg-Grunwald-Žalgiris in 1410, in: The Military Orders, vol. 5: Politics \& Power, ed. P. Edbury, Aldershot 2012, S. 329-336. Eine übersichtliche Darstellung der Söldnerfrage im Lichte der politischen Ereignisse bietet auch die Einleitung zum zweiten Band der Soldbuchedition: Das Soldbuch des Deutschen Ordens 1410/1411, Teil II: Indices mit personengeschichtlichen Kommentaren (Veröffentlichungen aus den Archiven Preußischer Kulturbesitz 23/II), bearb. v. S. Ekdahl, Köln- Weimar-Wien 2010, S. 1-12. (Zu Teil I siehe Anm. 47). Siehe ferner die in Anm. 15 und 18 angeführte Literatur.

14 Siehe beispielsweise Jóźwiak, Spionage (wie Anm. 12), S. 195 (allerdings ohne Hinweis auf die Provenienz der Behauptungen); J. Szymczak, W 600-lecie Wielkiej wojny z zakonem krzyżackim (od Eęczycy 17 VII 1409 do Torunia 1 II 1411), in: Na znak świetnego zwycięstwa (wie Anm. 2), Bd. I, S. 13-40, hier $23 \mathrm{f}$. 
Mai 1410 verhängten Werbungsstopps für Söldner, der zwei Wochen später aufgehoben werden musste, unterstreichen. ${ }^{15}$ Bedingt war diese Maßnahme durch die mit König Sigmund von Ungarn sowie mit dem polnischen König Jagiełło und dem litauischen Großfürsten Witold (lit. Vytautas) Mitte April in Käsmark (ung. Késmárk) vereinbarten, aber nie zustande gekommenen Friedensverhandlungen in Thorn am 17. Juni. ${ }^{16}$ Nur der Hochmeister mit seiner Entourage und einem Teil des Ordensheeres sowie eine ungarische Gesandtschaft ohne den König sind dort erschienen. Der Deutsche Orden hat dadurch eine schwere diplomatische Niederlage mit katastrophalen Folgen erlitten, denn erstens hatte Ulrich von Jungingen wegen der vorgesehenen Verhandlungen auf einen von ihm geplanten Überraschungsangriff auf Polen am 1 . Juni verzichten müssen ${ }^{17}$, und zweitens ließen sich wegen des Werbungsstopps große Söldnerscharen aus Böhmen und Mähren von Jagiełło anwerben. ${ }^{18}$ Sie hatten zunächst in den Dienst des Ordens treten wollen, waren aber aus den genannten Gründen abgewiesen worden und gingen deshalb aus finanziellen Erwägungen zu den Polen über. Außerdem kamen einige tausend Söldner durch den zweiwöchigen Werbungsstopp zu spät nach Preußen, um an der Feldschlacht am 15. Juli im Heer der Ordensritter teilnehmen zu können. ${ }^{19}$ So wurde einerseits die Streitmacht des Deutschen Ordens geschwächt, andererseits die der Polen und Litauer entsprechend gestärkt. Großfürst Witold hat

15 S. Ekdahl, W przededniu bitwy grunwaldzkiej. Dyplomacja i werbowanie żotnierzy zacięznych w czerwcu $1410 \mathrm{r}$., in: Wojna, pamięć, tożsamość. O bitwach i mitach bitewnych, hrsg. v. J. M. Piskorski, Warszawa 2012, S. 81-89 (Text) und 169-175 (Fußnoten) mit den dort angeführten Literaturhinweisen. Auf S. 80 ebd. eine Karte über den Aufmarsch der Heere: „Trasa marszu armii na pole bitwy pod Grunwaldem (według Svena Ekdahla, 2012)“. Über den Solddienst für den Deutschen Orden unterrichtet S. Ekdahl, The Teutonic Order's Mercenaries during the ,Great War' with Poland-Lithuania (1409-11), in: Mercenaries and Paid Men. The Mercenary Identity in the Middle Ages. Proceedings of a Conference held at University of Wales, Swansea, $7^{\text {th }}-9^{\text {th }}$ July 2005 (History of Warfare 47), ed. John France, Leiden, Boston 2008, S. 345-361. Siehe ferner Uwe Tresp, Söldner aus den Ländern der Böhmischen Krone in den Kriegen zwischen dem Deutschen Orden und Polen-Litauen zu Beginn des 15. Jahrbunderts, in: Tannenberg-Grunwald-Žalgiris (wie Anm. 12), S. 135-158, hier 149. Vgl. hierzu auch G. Vercamer, Die Freien im Deutschordensland Preußen als militärischer Rückhalt Ende des 14. - Anfang des 15. Jahrhunderts, in: Tannenberg-Grunwald-Žalgiris (wie Anm. 12), S. 175-197, hier 186.

16 Erstmalige ausführliche Analyse bei Ekdahl, Diplomatie und Söldnerwerbung (wie Anm. 13). Weitere Literatur in Anm. 13 und 15, u. a. Ekdahl, Politics, Diplomacy and the Recruitment of Mercenaries (wie Anm. 13).

17 Ebd. und beispielsweise Ekdahl, Die Söldnerwerbungen (wie Anm. 9), S. 101; ders., W przededniu (wie Anm. 15).

18 S. Ekdahl, Polnische Söldnerwerbungen vor der Schlacht bei Tannenberg (Grunwald), in: Non sensistis gladios. Studia ofiarowane Marianowi Gtoskowi w 70. rocznice urodzin, hrsg. v. O. Ławrynowicz, J. Maik, P. A. Nowakowski, Łódź 2011, S. 121-134.

19 Hierzu vor allem die grundlegende Untersuchung von S. Ekdahl, Kilka uwag o księdze żotdu 
übrigens mit der beachtlichen Summe von 20000 Schock Groschen zum Erfolg der polnischen Werbungen beigetragen. ${ }^{20}$ Wichtig ist in diesem Zusammenhang, dass Jagiełło es verstanden hat, mit verlockenden finanziellen Zusagen die Söldner auf seine Seite zu ziehen, denn er hat im Gegensatz zum Hochmeister großzügig Schadensersatz für verlorene Pferde und Ausrüstung versprochen. ${ }^{21}$ Das konnte den auf finanziellen Gewinn bedachten Söldnern nicht gleichgültig sein, und so wuchs ihre Anzahl im Heer der Unionsreiche beträchtlich. Die alte Behauptung von Oskar Halecki, „the Poles could only enlist a small number of Czech mercenaries", die auch in der litauischen Forschung vertreten wird, ${ }^{22}$ hat sich als unzutreffend erwiesen. ${ }^{23}$

Nach den Ausführungen von Jan Długosz in den Annales wurde auf einer Reichsversammlung in Kraków Ende April 1410 beschlossen, umfassende Werbungen durchzuführen. ${ }^{24}$ Es wurde betont, dass man durch sie die Söldner davon abhalten würde, zum Feind zu ziehen und dadurch dessen Kampfstärke zu vermehren. Auch meinte der damalige Marschall von Polen, Zbigniew von Brześć, dass fremde Hilfstruppen vorzuziehen seien, weil sie im Falle des Sieges nicht mit den Mitteln des polnischen Reiches, sondern denen der Feinde befriedigt werden könnten. Im Falle einer Niederlage aber „würde es weder solche geben, die Ansprüche erheben würden, noch solche, an die man sich seiner Ansprüche halber wenden könnte“.25

Diese von Długosz wiedergegebene Aussage des polnischen Marschalls ist hochinteressant und stützt meine Ansicht, dass Jagiełło sozusagen einen Wechsel auf die Zukunft gezogen hat. Er hat meines Erachtens alles auf eine Karte gesetzt, um eine Feldschlacht zu gewinnen und danach - was ebenso wichtig erschien die Hauptburg des Deutschen Ordens einzunehmen, denn dort befand sich der sehr große und von Mythen umwobene Schatzkammer (Tresel) der Ordensritter.

Zakonu Krzyżackiego z okresu „Wielkiej Wojny” 1410-1411, Zapiski Historyczne 33 (1968), 3 , S. 111-130. Siehe auch die in Anm. 13, 15, 18 angeführte Literatur.

20 Ekdahl, Polnische Söldnerwerbungen (wie Anm. 18), S. 124 (mit Quellenbeleg: OBA 1288).

21 Ebd. und Ekdahl, W przededniu (wie Anm. 15), S. 25. Die große Bedeutung der Schadensersatzfrage für die Söldner beider Seiten beleuchtet S. Ekdahl, Verträge des Deutschen Ordens mit Söldnerführern aus den ersten Jahrzehnten nach Grunwald, Questiones Medii Aevi Novae 11 (2006) (Arms and Armour, hrsg. v. J. Szymczak), S. 51-95. Vgl. Anm. 54.

22 M. Jučas, The Battle of Grünwald, ed. J. Everatt, M. Šapoka, Vilnius 2009, S. 54: „Jogaila and Vytautas could not hope for any military or political support from European leaders. They had to be content with only their own forces and the few mercenaries they managed to hire".

23 Ekdahl, Polnische Söldnerwerbungen (wie Anm. 18), S. 121, 129.

24 Ebd., S. 123 unter Hinweis auf Jan Długosz, Annales seu Cronicae incliti Regni Poloniae. Libri X et XI (1406-1412), ed. M. Plezia (et al.), Varsaviae 1997, S. 57 f.

25 Ebd. 
Schon vor 30 Jahren schrieb ich dazu, dass im Herbst 1410 die von Jagiełło ,angeworbenen Söldner drohten, wegen Zahlungsunfähigkeit des Königs das Heer zu verlassen; es war den Polen nämlich nicht gelungen, wie erhofft, Marienburg mit dem Ordensschatz zu erobern“. ${ }^{26}$ Mit jenem Geld hätte er problemlos alle seine Söldner entlohnen und seine großzügigen Versprechungen hinsichtlich des Schadensersatzes einhalten können. Die Eroberung der Hauptburg des Deutschen Ordens war im Hinblick auf die durch die vielen besoldeten Hilfstruppen entstandenen und im weiteren Verlauf des Krieges zu erwartenden hohen Ausgaben ein wichtiges Ziel des Feldzuges, ${ }^{27}$ auch wenn viele polnische Kollegen mit einer idealisierten oder romantischen Betrachtungsweise von Jagieło als rex iustus (in Anlehnung an Długosz) eine solche realistische, ich möchte sagen „materialistische", Deutung der Geschehnisse gewiss ablehnen werden. ${ }^{28}$

Als Stütze für meine Behauptung können etliche Quellenbelege angeführt werden, obwohl sie nicht von polnischer Seite stammen. In dem Aufruf des Reichsvikars und ungarischen Königs Sigmund von Luxemburg vom 20. August 1410 zur Unterstützung des durch die Schlacht bei Tannenberg/Grunwald sehr geschwächten Ordens heißt es bezeichnenderweise, dass dessen Feinde die Marienburg belagerten, in quo thesaurus eorum habetur et cor terre existit. ${ }^{29}$ Man kann sich übrigens

26 S. Ekdahl, Die Schlacht bei Tannenberg 1410. Quellenkritische Untersuchungen, Bd. I: Einführung und Quellenlage (Berliner Historische Studien 8), Berlin 1982, S. 164; ders., Grunwald 1410. Studia nad tradycją i źródtami, Kraków 2010, S. 164: „[...] a zaciężni grozili porzuceniem armii z powodu kłopotów króla z wypłaceniem im żołdu, jako że Polakom nie udało się, wbrew oczekiwaniom, zdobyć Malborka wraz z tamtejszym skarbcem Zakonu“. Vgl. dazu K. Militzer, Die Geschichte des Deutschen Ordens, Stuttgart 2005, S. 145: „Ihm [König Władysław Jagiełło S. E.] war es nicht gelungen, die Burg zu erobern und den Ordensschatz an sich zu bringen, damit er seine Söldner bezahlen konnte, und hatte damit ein wichtiges Kriegsziel verfehlt" (vgl. auch die polnische Übers.: ders., Historia zakonu krzyżackiego, übers. v. E. Marszał, J. Zakrzewski, Kraków 2007, S. 209).

27 Deshalb wurde im polnischen Heer schweres Belagerungsgerät, wie große Kanonen und Bliden, mitgeführt. S. Ekdahl, The Siege Machines during the Baltic Crusades, Fasciculi Archaeologiae Historicae 20 (2007) (Les envahisseurs des temps médiévaux et leurs armes, hrsg. v. Tadeusz Poklewski-Koziełt), S. 29-51, hier 51. Die allergrößte Büchse konnte allerdings nicht geladen werden, weil kein Transportwagen das Gewicht zu tragen vermochte. Sie musste in Krakau zurückgelassen werden. Siehe dazu meine Rezension von Jan Szymczaks Buch Początki broni palnej w Polsce (1383-1533), Militärgeschichtliche Zeitschrift (67) 2008, 1, S. 202-204, hier S. 203.

28 Als ich einmal vor Jahren einem polnischen Kollegen (kein Historiker) meine Ansicht erzählte, wies er sie empört zurück, denn solch niedrige Beweggründe seien dem polnischen König nicht zuzutrauen. Diese Auffassung hat er später wiederholt: „Es war nicht der Wille des Königs, den Ordensschatz zu rauben, wie S. Ekdahl meint, und Jagiełło hatte als König eines großen Staates nicht die Mentalität eines Raubritters" (Manuskriptentwurf vom Mai 2012). 
gut vorstellen, dass es gerade für Sigmund einem Albtraum gleichgekommen wäre, hätten die Polen den Schatz der Ordensritter in ihre Hände bringen können, denn gerade er war der größte Nutznießer desselben. ${ }^{30}$ Sicherlich nicht zuletzt aus diesem Grund hat er die Belagerten vehement zum Ausharren aufgefordert und Hilfe angekündigt. ${ }^{31}$ Es gibt aber auch weitere Behauptungen, die in dieselbe Richtung zeigen. Am 14. Dezember 1410 verschickte der neue Hochmeister Heinrich von Plauen ein langes Rundschreiben an Fürsten und Herren, in dem ebenfalls auf die Bedeutung des Ordensschatzes im strategischen Denken Jagiełłos hingewiesen wurde. Darin wird betont, dass der König von Polen die Eroberung der Marienburg in der Absicht anstrebte, ap her das hus g[e]winnen mochte mit des ordens schatcze. ${ }^{32}$ Schon am 22. Juli, also eine Woche nach der Schlacht, hatte von Plauen in einem auf der Marienburg verfassten Rundschreiben hervorgehoben: In qua obsidione vero tocius Ordinis potestas et potentia consistunt. ${ }^{33}$ Weniger aussagekräftig, aber doch ebenfalls von Interesse, sind einige Chroniknotizen. ${ }^{34}$

v. T. Hirsch, M. Töppen, E.Strehlke, Leipzig 1866, S. 403 f., das Zitat auf S. 403. Hierzu Ekdahl, Die Schlacht (wie Anm. 26), S. 181 f.; ders., Grunwald 1410 (wie Anm. 26), S. 179 f. Vgl. Z. H. Nowak, Internationale Schiedsprozesse als ein Werkzeug der Politik König Sigismunds in Ostmittel- und Nordeuropa 1411-1425, Blätter für deutsche Landesgeschichte (111) 1975, S. 172-188, hier 176.

30 Man vergleiche dazu die kritische Bewertung Sigmunds durch den preußischen Chronisten Posilges Fortsetzer im Jahr 1412: [...] was gutes willen der herre koning von Ungern vor bewyset hatte, das tat her alles dorumb, das der ordin im gut und geld sulde habin gegebin (SS. rer. Pruss. III, S. 330).

31 Spätestens am 8. August war die Kunde von der Niederlage des Deutschen Ordens in der ungarischen Hauptstadt Buda eingetroffen, denn an jenem Tag schrieb Sigmund einen Brief mit einer Mahnung an Landvolk, Ritter und Knechte in Preußen und an die Stadt Danzig, am Deutschen Orden festzuhalten. Druck des Briefes bei Z. H. Nowak, Polityka pótnocna Zygmunta Luksemburskiego do roku 1411 (Roczniki Towarzystwa Naukowego w Toruniu 69, 1), Toruń 1964, S. 131 f. Bei Posilges Fortsetzer heißt es über die Belagerung: Ouch qwomen brife von dem konige von Ungern uf das hus, sy suldin sich vaste haldin, her welde sy wol entsetczin (SS. rer. Pruss. III, S. 321).

32 Zitat nach OBA 1418. Druck des Rundschreibens in: Jahrbücher Johannes Lindenblatts oder Chronik Johannes von der Pusilie, Officials zu Riesenburg, hrsg. v. J. Voigt, F. W. Schubert, Königsberg 1823, Beilage III, S. 395-399, die Erwähnung des Ordensschatzes auf S. 396.

33 M. Biskup, Echa bitwy grunwaldzkiej i oblężenia Malborka w niemieckiej gatęzi zakonu krzyżackiego w lecie 1410 roku, Komunikaty Mazursko-Warmińskie 1983, 4 (162), S. 455-460, hier 456. Die große Belagerung der Marienburg begann zwar erst am 25. Juli, aber sie war vorauszusehen und wurde von Heinrich von Plauen schon jetzt als Faktum hingestellt. Kleinere Abteilungen des polnisch-litauischen Heeres könnten allerdings vorausgeschickt und gemeint gewesen sein (Biskup (wie oben), S. 456).

34 Nach der Fortsetzung von Detmars Lübischer Chronik in der Hamburger Handschrift zogen die Könige von Krakau und Litauen mit unmaten vele volkes vor de Marienborch, de to wynnende dor des groten schattes willen, de en was vormeldet (SS. rer. Pruss. III, S. 406), und in dem 
Was hat nun dies alles mit dem Thorner Frieden zu tun? Ich meine, wie beispielsweise schon Stefan M. Kuczyński in seinem Buch „Wielka wojna“, dass man Jagiełło und seinen Verhandlungsführern unrecht tut, wenn man ihnen vorwirft, den großen Sieg bei Grunwald sozusagen verspielt zu haben. ${ }^{35}$ Diese vielen Kritiker, von Długosz angefangen, der übrigens dem litauischen Großfürsten eine große Mitschuld zuschiebt ${ }^{36}$, sind der Ansicht, dass größere Gebietsabtretungen, wie die Pommerellens ${ }^{37}$, gerechtfertigt gewesen wären und dass die Verhandlungen auf polnischer Seite nicht kraftvoll genug geführt worden seien. Die Verpflichtung zur Zahlung von 100000 Schock Prager Groschen (d.h. sechs Millionen Groschen im Gegenwert von 10000 guten Kriegspferden), die Jagiełło dem Hochmeister in einem Verpflichtungsbrief vom 31. Januar 1411, also einen Tag vor dem Abschluss des Thorner Friedens, abgerungen hat, wird von den Kritikern als zu geringe Ausbeute des Sieges betrachtet. ${ }^{38}$

Wir erinnern uns daran, dass das Fehderecht, das Kriegsrecht, die Freilassung aller Gefangenen auf beiden Seiten ohne Lösegeld als Voraussetzung für einen gültigen Friedensschluss festlegte. ${ }^{39}$ Deshalb heißt es in der Thorner Friedensurkun-

Chronicon Bohemie von 1344-1411 heißt es zum Jahr 1410, dass der König von Polen ivit cum gravi multitudine ante castrum Marigenborch, ubi Prutheni habuerunt thesaurum ipsorum, et jacuit ibi bene per decem septimanas, sed tamen castrum non valuit acquirere (ebd., S. 429). In einem Breve Chronicon rerum suo tempore gestarum 1396-1418 eines anonymen bayerischen Chronisten wird der Ordensschatz ebenfalls erwähnt (ebd., S. 418). Nach den Ersten Pleskauer Annalen habe sich das Heer auf dem Marsch zur Marienburg befunden, als es auf die gesamte Streitmacht des Hochmeisters gestoßen sei: Ekdahl, Die Schlacht (wie Anm. 26), S. 155; ders., Grunwald 1410 (wie Anm. 26), S. 156 f. In seinem Reisebericht nennt Guilbert de Lannoy 1413 die Marienburg, qui est ville et chastel tres fort, ou quel gist le tresor, la force et tout le retrait de tous les seigneurs de Prusse (SS. rer. Pruss. III, S. 444).

35 S. M. Kuczyński, Wielka wojna z Zakonem Krzyżackim, Warszawa ${ }^{5} 1987$, S. 620-623 (in der deutschen Zusammenfassung).

36 Długosz, Annales, lib. X/XI (wie Anm. 24), S. 177 f. Die Überschrift des Abschnitts lautet: Wladislaus rex consultore Alexandro duce magno Lithuanie Polonis turpe et dampnosum cum Pruthenis perpetuum init foedus.

37 Die Eroberung Pommerellens durch den Deutschen Orden 1308/1309 war in Polen nie vergessen worden. In einem Bericht vom 4. August 1420 mit Nachrichten aus Polen und über Witold heißt es dazu: Die Polen hätten gesagt, is, das sie me kriegen wurden mit deme Orden, das welden sie thun umbe das landt czu Pomern, das von alders czur Crone kegen Polan gehort hat (OBA 3224).

38 Hierzu Kuczyński, Wielka wojna (wie Anm. 35), S. 536-550, sowie Kapitel VI in dem Buch Wojna Polski i Litwy (wie Anm. 3), S. 694-747 mit Beiträgen von S. Szybkowski, A. Szweda und K. Kwiatkowski. Siehe auch die oben in Anm. 7 genannten Tagungsaufsätze.

39 Ekdahl, Die Schlacht (wie Anm. 26), S. 169-179, unter Hinweis auf O. Brunner, Land und Herrschaft. Grundfragen der territorialen Verfassungsgeschichte Österreichs im Mittelalter, Darmstadt 1973 (unveränderter reprografischer Nachdruck der 5. Aufl., Wien 1965), S. 106; Ekdahl, Grunwald 1410 (wie Anm. 26), S. 169. 
de vom 1. Februar 1411: Item quod omnes captivi, qui in hac guerra sunt captivati, a captivitatibus ipsorum de ambabus partibus fiant quiti, liberi et soluti sine fraude et quod omnes depactaciones et exacciones eorundem captivorum, que nondum sunt exacte, deleantur et in nichilum redigantur. Et si aliqui pro ipsorum depactacione cauciones fideiussorias fecerunt aut eciam litterales, omnes omnino anullentur et fideiussores sint liberi dolo et fraude quibuslibet proculmotis. ${ }^{40}$ Durch den am Tag zuvor geschriebenen Verpflichtungsbrief konnte diese Bestimmung umgangen werden und Jagiełło somit rechtlich korrekt ein Lösegeld für seine vielen Gefangenen und für die Rückgabe noch besetzter Burgen erhalten. Das ist die einfache Erklärung dafür, dass die 100000 Schock Groschen nicht in der Friedensurkunde erwähnt werden. ${ }^{41}$

Der genannte Verpflichtungsbrief wurde 1979 als Abschrift in einem Folianten im Ordensarchiv von dem amerikanischen Historiker Markian Pelech gefunden und von ihm in „Preußenland“ herausgegeben. ${ }^{42}$ Dass der Orden die letzten der dort festgelegten vier Zahlungstermine nicht einhalten konnte oder wollte, ist eine andere Frage. Im Jahr 1411 erhielten die Polen 25000 Schock am 10. März und 20000 Schock am 25. Juni, während die verbliebene Schuldsumme erst später beglichen wurde, letztmalig am 18. Januar $1413 .{ }^{43}$

Ich frage nun: Was hätte der König in der Lage, in der er sich Anfang des Jahres 1411 befand, anderes tun können, als auch Geld als Gegenleistung für den Frieden zu fordern? Gewiss hat er sich, wie alle Polen, einen größeren Zugewinn an

40 Die Staatsverträge des Deutschen Ordens in Preußen im 15. Jahrhundert, Bd. I (1386-1437) hrsg. v. E. Weise, Marburg ${ }^{2} 1970$, S. 86.

41 Dieser Gesichtspunkt ist von Klaus Neitmann in seinen Bemerkungen zum 1. Thorner Frieden nicht erörtert worden. K. Neitmann, Die Staatsverträge des Deutschen Ordens in Preußen 1230-1449. Studien zur Diplomatie eines spätmittelalterlichen deutschen Territorialstaates (Neue Forschungen zur Brandenburg-Preußischen Geschichte 6), Köln-Wien 1986, S. $162-$ -165 und passim. Vgl. dazu ders., Vom „ewigen Frieden“. Die Kunst des Friedensschlusses zwischen dem Deutschen Orden und Polen-Litauen 1398-1435, in: Tannenberg-Grunwald-Žalgiris (wie Anm. 12), S. 201-209, vor allem S. 201, Anm. 1.

42 M. Pelech, Der Verpflichtungsbrief des Hochmeisters Heinrich von Plauen bezüglich der Bezahlung von 100000 Schock Böhmischer Groschen an den König von Polen vom 31. Januar 1411, Preußenland 17 (1979), 4, S. 55-64.

43 Die Staatsverträge (wie Anm. 40), S. 90, sowie die Ausführungen von Pelech, Der Verpflichtungsbrief (wie Anm. 42), S. 56-58, und ders., W sprawie okupu za jeńców krzyżackich z wielkiej wojny (1409-1411), Zapiski Historyczne 52 (1987), 1, S. 131-152; Zapiski Historyczne 52 (1987), 2, S. 95-112. Vgl. S. Jóźwiak, Wajennalapalonnyja Njameukaga ordzna bitwy pad Karanovam 1410 g. [Deutschordens-Gefangene in der Schlacht bei Koronowo 1410], Belarusian Historical Review 17 (2010), 1-2, S. 65-88, hier 82-87. Siehe demnächst auch den Konferenzbeitrag von A. Szweda, Wie wurden die im 1. Thorner Frieden festgehaltenen Vertragsbedingungen im weiteren Verlauf der Beziehungen zwischen Polen und dem Deutschen Orden eingehalten? (wie Anm. 7). 
Territorium als die erfolgten Zugeständnisse des Ordens in der Friedensurkunde gewünscht ${ }^{44}$, aber sowohl die finanzielle, als auch die politische und militärische Lage vereitelte solche Pläne. Wichtiger war angesichts der Umstände das Anliegen, die vielen noch nicht entlohnten Söldner zu beruhigen und Geld anstatt mehr Land zu fordern. Ein großer Sieg war zwar errungen worden, jedoch war das in der Krakauer Reichsversammlung Ende April 1410 ausgesprochene Ziel mit der Anwerbung von Söldnern nicht zur vollen Zufriedenheit erreicht worden, denn es fehlten jetzt die Mittel zu ihrer Entlohnung. ${ }^{45}$ Das Vabanquespiel mit dem Ziehen eines Wechsels auf die Zukunft war nur zum Teil gelungen, die Eroberung der Marienburg mit dem Ordensschatz war trotz der acht Wochen langen Belagerung gescheitert. Damit stand Jagiełło vor einem gewaltigen finanziellen Problem, mit dem er während der ersten Tage nach der Schlacht bei Grunwald sicherlich nicht gerechnet hatte, das ihn aber während des ganzen Krieges verfolgen sollte. Übrigens war die Lage in dem vier Jahre später begonnenen sog. „Hungerkrieg“ mit dem Deutschen Orden ähnlich, denn der konyng schit us dem lande mit syme grosin schadin: her hatte synen soldenern nicht zcu lonen, her hatte sy als getrost off den ordin; ader is ging ym nicht noch synem willin. ${ }^{46}$ Jagiełło hat also 1414 seine Taktik wiederholt und auch diesmal die Söldner mit Verheißungen auf zukünftigen finanziellen Gewinn in Preußen vertröstet.

Heinrich von Plauen dagegen ist es durch die erfolgreiche Verteidigung der Hauptburg gelungen, die Karten neu zu mischen und eine noch verheerendere Niederlage des Ordens zu verhindern. Im Falle eines Verlustes der finanziellen Rücklagen auf der Marienburg hätten nämlich die Ordensritter die Forderungen der vielen eigenen Söldner nicht begleichen können und Preußen wäre in den totalen Ruin mit unvorhersehbaren Konsequenzen getrieben worden. Nun konnte der Krieg im Herbst 1410 trotz einiger weiterer schwerer Niederlagen des Ordens mit Hilfe von Söldnern und „Gästen“ weitergeführt und ohne substantielle Gebietsabtretungen im eigentlichen Ordensland im Thorner Frieden beendet werden. Die gewaltigen Summen, welche die Soldzahlungen der Ordensritter verschlangen, sind vor allem im Soldbuch von 1410/1411 verzeichnet worden. ${ }^{47}$ Jürgen Sar-

44 Über die alten Streitfragen betreffend die Burgen Driesen (Drezdenko) und Zantoch (Santok), auf die Polen Anspruch erhob, sollten Schiedsgerichte entscheiden; Die Staatsverträge (wie Anm. 40), S. 87.

45 Siehe die Aussage des vom Deutschen Orden im Herbst 1410 gefangengenommenen Söldners Wulffhart (wie Anm. 51).

46 SS. rer. Pruss. III, S. 347.

47 Das Soldbuch des Deutschen Ordens 1410/1411. Die Abrechnungen für die Soldtruppen. Mit ergänzenden Quellen, Tl. I: Text mit Anhang und Erläuterungen, bearb. und hrsg. v. S. Ekdahl (Veröffentlichungen aus den Archiven Preußischer Kulturbesitz 23/I), Köln-Wien 1988. Zu Teil II der Edition mit Indices und personengeschichtlichen Kommentaren siehe Anm. 12. 
nowski hat einmal die Mühe auf sich genommen, die Kosten für die besoldeten Hilfstruppen des Ordens während des gesamten Krieges 1409 bis 1411 zu berechnen, und ist dabei auf eine Summe von 226000 preußische Mark gekommen. ${ }^{48}$ Es gab aber auch viele Söldner, die im Soldbuch nicht verzeichnet sind, weshalb die reale Ziffer noch höher liegt. ${ }^{49}$

Weitere Quellen untermauern das Gesagte. Wichtig für die Beurteilung der finanziellen Schwierigkeiten der Polen im Herbst 1410 sind beispielsweise die Aussagen eines von den Ordenstruppen gefangengenommenen böhmischen Söldners namens Wulffhart, bei dem es sich nach Krzysztof Kwiatkowski um einen Ritter Wolfhart von der Horke aus der Oberlausitz handeln dürfte. ${ }^{50}$ Er wird in einem Brief des Hauskomturs von Schwetz an den stellvertretenden Hochmeister Heinrich von Plauen vom 3. November erwähnt. ${ }^{51}$ Den Angaben dieses Söldners zufolge befänden sich noch Böhmen mit 4000 Pferden beim polnischen König. Das Wort „noch“ macht klar, dass es ursprünglich mehr gewesen sein müssen, dass viele aber bereits ihren Dienst quittiert hatten. Die Verbliebenen wären auch gern weggezogen, heißt es, aber sie konnten es nicht tun, weil sie ihren Sold noch nicht erhalten hätten. Der König habe sie gebeten, bis zur Fassnacht des nächsten Jahres, d.h. bis zum 24. Februar 1411, bei ihm zu bleiben; dann würde Witold wieder ins Land kommen und ihnen würde Sold und Schadensersatz ausgezahlt werden. Um Geld für die Soldzahlungen aufzubringen, würde jedes polnische Dorf ein Schock Groschen aufbringen, und außerdem hätten die Landherren versprochen, Geld beizusteuern.

In seinem Brief fügte der Hauskomtur hinzu, dass Wulffhart der Meinung sei, er könne böhmische Söldner mit etwa 500 oder 1000 Pferden aus dem polnischen Heer dem Ordensheer zuführen. Das Problem dabei sei nur, dass sie sich um die Versorgung der Pferde mit Futter im Ordensland Sorge machten, denn sie liebten ihre Pferde.

48 J. Sarnowsky, Die Wirtschaftsführung des Deutschen Ordens in Preußen (1382-1454) (Veröffentlichungen aus den Archiven Preußischer Kulturbesitz 34), Köln-Weimar-Wien 1993, S. 406; ders., Wirtschaftliche Aspekte der Geschichte der Kriege am Beginn des 15. Jahrhunderts, in: Tannenberg-Grunwald-Žalgiris (wie Anm. 12), S. 123-134, hier S. 127.

49 Literatur wie in Anm. 13 und 15, unter anderem Ekdahl, W przededniu (wie Anm. 15), S. 87 f. Insgesamt befanden sich bei Tannenberg rund 6400 Söldner auf der Seite des Deutschen Ordens, von denen nicht alle im Soldbuch verzeichnet sind. Auch diejenigen, die zu spät gekommen sind, um an der Schlacht teilnehmen zu können - immerhin einige Tausend - haben ihren vereinbarten Sold erhalten. Für sie hat es z. T. gesonderte Abrechnungen gegeben, die nicht erhalten geblieben sind.

50 Wojna Polski i Litwy (wie Anm. 3), S. 632 f., vor allem Anm. 453 ebd.

51 Druck des Briefes bei Ekdahl, Polnische Söldnerwerbungen (wie Anm. 18), Quellenanhang, S. $129 \mathrm{f}$. 
Diese wichtige Quelle bestätigt also voll und ganz die prekäre finanzielle Lage Jagiełtos vor dem Abschluss des Thorner Friedens. Nach einem anderen Bericht vom 8. Januar 1411 befanden sich zu Beginn des Jahres nur noch wenige „Gäste "im Heer des Königs. ${ }^{52}$ Angesichts dieser Umstände konnten Jagiełło und seine Berater keine härteren Forderungen während der Verhandlungen im Januar 1411 durchsetzen und mussten sich mit dem Verpflichtungsbrief ohne Gebietsabtretungen im eigentlichen Ordensland zufrieden geben.

Abschließend soll als Kontrast zur finanziellen Bedrängnis der Polen die Lage auf Seiten der Ordensritter kurz erwähnt werden. Der Orden hat im Herbst 1410 mit Hilfe seines Münz- und Silberschatzes auf der Marienburg Söldnerwerbungen in großem Stil fortsetzen können und war zum Schluss, wie Heinrich von Plauen in seinem nach England gesandten Bericht vom ersten November 1412 betont, mit sulcher macht besammelt, das her dem von Polan wol czu starg was..$^{53}$ Auf Drängen der "Gäste“ des Ordens und angesichts der Tatsache, dass Jagiełło nun wieder ein großes Heer der Heiden - worunter wir in der Terminologie des Ordens die Truppen Witolds zu verstehen haben - nach Polen geholt hatte, hätte sich der Orden jedoch unverhofft und notgedrungen zum Einlenken gezwungen gesehen: Semliche dirbitunge tat her [d. h. der polnische König] so lang, bis das her abir [nochmals] syne heiden [d. h. Witolds Heer] mechtichlichin czu im brachte, also das der Orden off die czit ungehoft gedrungen wart, eyne berychtigunge mit im anczugeen..$^{54}$

So mussten beide Seiten am 1. Februar 1411 mit gemischten Gefühlen aufeinander zugehen. Der Konflikt schwelte deshalb weiter und der erste Thorner Frieden wurde trotz der mit vielen Siegeln versehenen schönen Pergamenturkunden kein „ewiger“.

52 Brief des Bürgers David Rosenfeld an Hochmeister Heinrich von Plauen; siehe unten: Quellenanhang.

53 OBA 1737, f. 191r.

54 Ebd., f. 191v. Siehe auch OBA 1481 und 1482 (Klageschriften des Hochmeisters vom 15. Januar 1411), sowie die 1412 verfasste Werbunge des huskomthurs von Thorun an die fursten und herren zu dutschen landen etc., in: Codex epistolaris Vitoldi magni ducis Lithuaniae 1376-1430, ed. A. Prochaska, pars 1, Cracoviae 1882, Nr. 498, S. 233-246, hier 238 f. Vgl. Ekdahl, $W$ przededniu (wie Anm. 15), S. 88 f. mit Anm. 63 aufS. 174 f. Am 13. Januar 1411 hat der Hochmeister den monatlichen Sold vieler Söldner von 22 auf 24 Gulden pro Spieß erhöht und ihnen auch andere wichtige Vergünstigungen eingeräumt ([... steht in auch schaden, vor gefengknuß $[. .]$.$) ,$ um sie zum Verbleib im Ordensheer zu bewegen. K. Kwiatkowski, Neue Quellen aus dem Kreis des Deutschen Ordens zum Krieg von 1409-1411 (Teil I), Zapiski Historyczne 75 (2010), 4, S. 67-112, hier Nr. 29, S. 103. 


\section{QUellenanhang}

Kulm, 8. Januar 1411

Der Bürger David Rosenfeld an den Hochmeister [Heinrich von Plauen]: Nachrichten aus Polen über Aufgebote und Kriegsvorbereitungen. Der König wird in Leslau [Wtoctawek] erwartet.

GStA PK, XX. HA StA Kbg., OBA 1477.

Alte Signatur: Schieblade XX, Nr. 82.

Ausfertigung, Papier $(22 \times 30 \mathrm{~cm})$, mit sehr geringen Resten eines braunen Verschlusssiegels (Durchmesser: $20 \mathrm{~mm}$ ). Ohne Wasserzeichen. Beschädigt durch große Stockflecke, kleinere Löcher und Einrisse, jedoch ohne Textverlust.

Umseitig Adresse: Deme erenwirdigen mynem allirgnedigesten hirren hoemeister mit aller erenwirdekeit.

Williger dinst mit demutiger bevelunge zcuvor. Erenwirdiger liebir gnediger hirre, euwir erenwirdige gnade geruche wissen, das euwir dyner nulichin by mir gewest ist und worhafftiglichin hat gesagit, das sich der koning von Polan gros vorsammilt, als von eyginner macht mit im nemende alle syne ritter und knechte, als der man ist gesessin, us den steten die helffte, und die gebuwer von den dorffern, als sie sien gesessin, die czulowffen mit keulen und spyssen, als uns gerede angehorit, wendt her wenig geste by im hat. Und disse macht sich vorsammeln sal uff jeneseyt Lesselaw, und czu in sal komen der starost us Polan, und man richtet czu czu Lesselaw uff den koning, wendt her gewislich sal dar komen. Sundirlichin so sagit dirre selbie euwir dyner, das her vornomen habe von den Polan als sagemere, das her doch worhafftiglichin nicht kunde dirvorsschin, das Wythawt dem konnige czu holffe kome dorch die Masaw. Hirvon ich ouch euwirn gnaden keyne warheyt kann geschrebin. Ouch so ist dirre selbie euwir dyner itczundt wedir uff den verthen und wyl czihen in das polnissche heer all, solde her synen hals dorumme vorlysen, und wyl das heer und alle seyne macht eygintlichin besehen und gentczlichin dirvorssschen. Dis euwirn gnaden ich dinstlichin vor worhafftige czeitunge schreybe.

Ouch, erenwirdiger gnediger hirre, so hat dirre selbie euwir dyner mit seynen ougen gesen sichtiglichin, das der koning in allen enden ken dem lande czu Prussen warthe hat bestalt czu halden tag und nacht dorch syne ritter und knechte. Hirnoch so mag sich euwir gnade wol richten.

Geben czum Colmen [dt. Kulm, poln. Chetmno] am donrstage noch der Heyligen Dry Koninge tage czwusschen vir und vunffen noch mittage anno $\mathrm{CCCC}^{\circ}$ undecimo.

David Rosenveldt, euwir unwirdiger dyner. 
